small diameter, which surmounts it, is exposed to the internal pressures, which produce liquefaction, while its outer walls only sustain the atmospheric pressure. The tube passes vertically through a metal shoulder-piece, so that all the phases of liquefaction can be followed by the naked eye. For greater security, it is well to surround this part of the apparatus with a larger cylinder filled with water.

The gas is compressed by a hydraulic pump, with the intervention of a layer of mercury. The apparatus is so simple, and so easy to operate without danger, that I hope it may be commonly employed in lectures and in laboratories, for repeating experiments of this kind.

\title{
LIQUEFACTION OF NITROGEN, HYDROGEN AND AIR.
}

Note by M. L. Catlletet. ${ }^{i}$

I have continued my experiments upon the liquefaction of gases, and I am happy to inform the Academy that $I$ have succeeded in liquefying nitrogen and atmospheric air. Hydrogen itself gives evidence of liquefaction, as I will presently show. I submit some details of my experiments:

Nitrogen.-Nitrogen, pure and dry, compressed by about 200 atmospheres at a temperature of $+13^{\circ}$, then suddenly set free, is condensed in the neatest manner. There is first formed a material like a pulverized liquid, in drops of an appreciable volume; then this liquid gradually disappears from the walls towards the centre of the tube, forming a sort of vertical column in the very axis of the tube. The total duration of the phenomenon is about three seconds.

These appearances leave no doubt as to the true character of the phenomenon. I first made the experiment at home, at a temperature of $-29^{\circ}$, and I repeated it yesterday, Dec. 30th, a large number of times, in the laboratory of the Normal School, in the presence of many savants and members of the Academy, among whom I am happy to name, with his permission, the honored M. Boussingault.

${ }^{1}$ Comptes Rendus, Dec. 31st, 1877. 
Hydrogen.-Hydrogen has always been regarded as the most incoercible gas, because of its small density and the nearly complete agreement of its mechanical properties with those of perfect gases. It was, therefore, only with an extreme distrust of the result, that I decided to submit it to the same ordeals as determined the liquefaction of all the other gases.

In my first attempts I noticed nothing peculiar; but as often happens in the experimental sciences, the habit of observing phenomena ends in causing their signs to be recognized, in conditions under which they at first passed unnoticed. This was the case with hydrogen. In repeating the experiment, this very day, before MM. Berthelot, $H$. Sainte-Claire Deville, and Mascart, who authorize me to invoke their testimony, I observed indications of the liquefaction of hydrogen, under conditions of evidence which did not appear doubtful to any of the learned witnesses. The experiment was repeated many times. In operating with pure hydrogen, under a pressure of about 280 atmospheres, then suddenly set free, we saw the formation of an exceedingly fine and subtle mist, suspended in the whole length of the gas, and which suddenly disappeared. The production of this mist, notwithstanding its extreme subtlety, appeared incontestable to all the savants who saw the experiment to-day, and who took care to have it often repeated, in such ways as to leave no doubt of its reality.

Air.-Having liquefied nitrogen and oxygen, the liquefaction of air followed as a matter of course; but I thought it would be interesting to make a direct experiment, which, as might have been expected, succeeded perfectly. The air was first dried, and deprived of carbonic acid. Complete confirmation has thus been given to the accuracy of the views expressed by the founder of modern chemistry, Lavoisier, upon the possibility of reducing air to a state of liquidity, thereby producing materials endowed with new and unknown properties, views recalled so pertinently, at the last meeting, by our illustrious perpetual Secretary.

I beg, in conclusion, to express my deep thankfulness to M. Berthelot, and to my dear master, M. H. Sainte-Claire Deville, for all the encouragement that they have kindly given me, as well as for the generous hospitality which has always been shown me in the laboratory of the Normal School. 